\title{
Dietary modulation of the human gut microflora using prebiotics
}

\author{
Glenn R. Gibson \\ Microbiology Department, Institute of Food Research, Reading, UK
}

\begin{abstract}
The human colonic flora has both beneficial and pathogenic potentials with respect to host health. There is now much interest in manipulation of the microbiota composition in order to improve the potentially beneficial aspects. The prebiotic approach dictates that non-viable food components are specifically fermented in the colon by indigenous bacteria thought to be of positive value, e.g. bifidobacteria, lactobacilli. Any food ingredient that enters the large intestine is a candidate prebiotic. However, to be effective, selectivity of the fermentation is essential. Most current attention and success has been derived using non-digestible oligosaccharides. Types primarily being looked at include those which contain fructose, xylose, soya, galactose, glucose and mannose. In particular, fructose-containing oligosaccharides, which occur naturally in a variety of plants such as onion, asparagus, chicory, banana and artichoke, fulfil the prebiotic criteria. Various data have shown that fructo-oligosaccharides (FOS) are specifically fermented by bifidobacteria. During controlled feeding studies, ingestion of these prebiotics causes bifidobacteria to become numerically dominant in faeces. Recent studies have indicated that a FOS dose of $4 \mathrm{~g} / \mathrm{d}$ is prebiotic. To exploit this concept more fully, there is a need for assessments of (a) improved determination of the gut microbiota composition and activity; (b) the use of molecular methodologies to assess accurately prebiotic identities and develop efficient bacterial probing strategies; (c) the prebiotic potential of raw and processed foods; and (d) the health consequences of dietary modulation.
\end{abstract}

Gut flora: Prebiotics: Fermentation

Recent years have seen major changes in the manner in which clinicians view the biological roles of the human gastrointestinal tract. In particular, the colon is no longer thought of as an organ of, primarily, absorption and storage. The resident bacterial flora of the large gut comprises about $95 \%$ of the total cells in the body, representing up to $10^{12}$ cells/g dry weight faeces, and plays a major role in host nutrition and health. Much attention is now therefore being directed towards dietary components that affect the gut in such a manner that the activities of benign or health-promoting bacteria are stimulated, preferably at the expense of pathogens. In this context, one can identify important roles for prebiotics that have a very selective fermentation in the large gut.

\section{Human gut bacteriology}

Helicobacter pylori is one of the most intensively studied micro-organisms of recent times. The bacterium resides in the mucosal layer of the stomach and is thought to be involved in the aetiology of various gastric complaints. These include type B gastritis, stomach ulcers and even gastric carcinomas (Parsonnet et al. 1991; Dixon, 1992; Wyatt, 1992). The implications of identifying a transmissible form of cancer that may be both preventable and susceptible to treatment are enormous. Interest in $\mathrm{H}$. pylori has also markedly raised the profile of gastrointestinal microbiology such that the significance of the resident microflora is a contemporary research area.

The human large intestine is the most heavily colonized region of the digestive tract (Cummings \& Macfarlane, 1991). Through the process of fermentation, colonic bacteria are able to produce a wide range of compounds which have varying potential effects on gut physiology, as well as other systemic influences.

At least fifty different genera of bacteria reside in the colon, comprising several hundred individual species. The activities of colonic bacteria are affected by a number of physicochemical parameters, with the bacteria present having fluctuating activities in response to substrate availability, redox potential, $\mathrm{pH}, \mathrm{O}_{2}$ tension and distribution in the colon. Most human large intestinal micro-organisms have a strictly anaerobic metabolism, whilst numbers of facultative anaerobes are much lower than those of the obligate species. The numerically predominant anaerobes are bacteroides, bifidobacteria, eubacteria, clostridia, lactobacilli, Gram-positive anaerobic cocci, coliforms, methanogens and dissimilatory sulphate-reducing bacteria (Moore \& Holdeman, 1974; Finegold et al. 1983; Cummings \& Macfarlane, 1991).

In very general terms, intestinal bacteria may be divided into species that are either harmful or beneficial towards host welfare. Pathogenic effects include diarrhoea, infections, liver damage, carcinogenesis and intestinal 


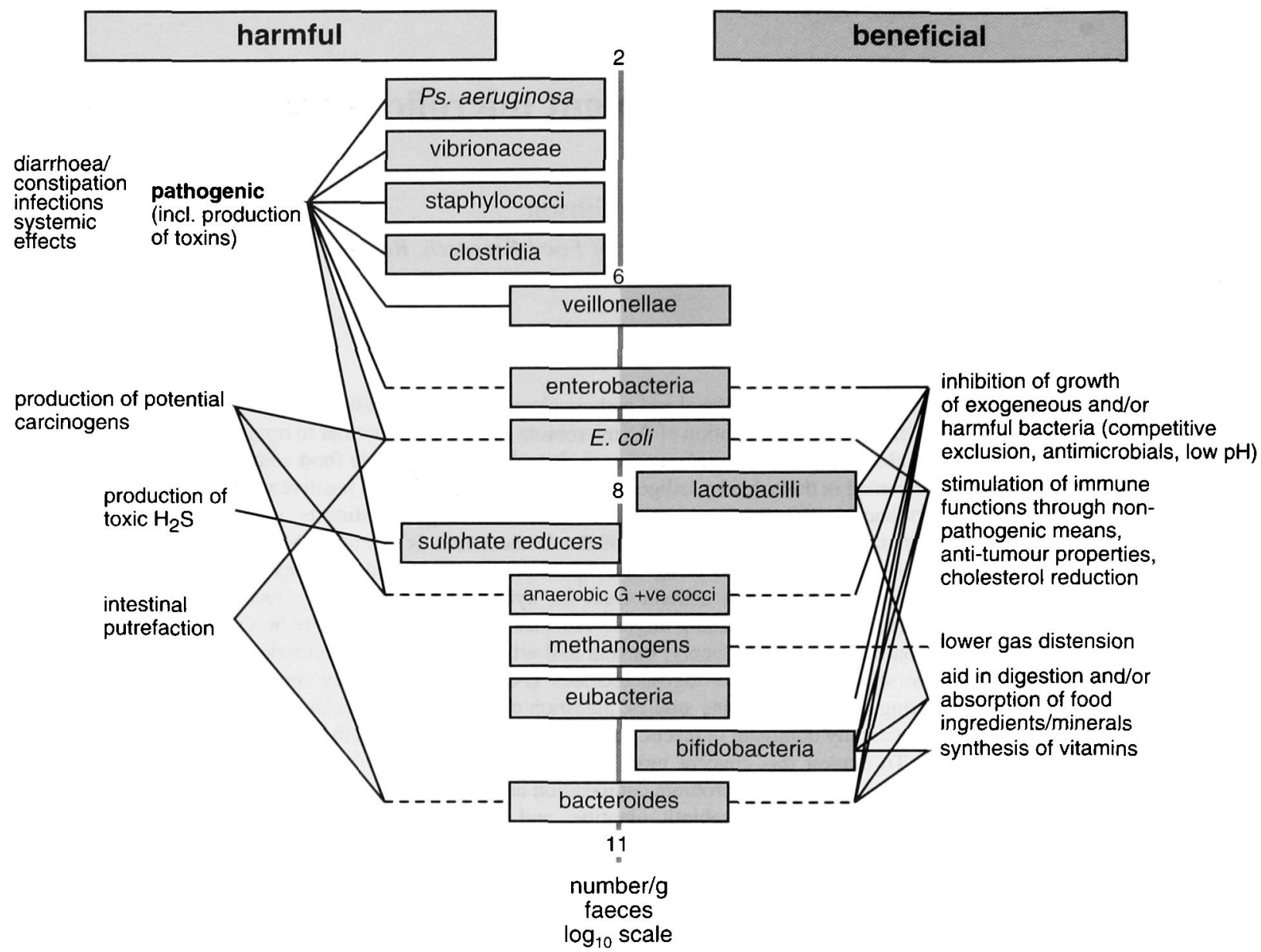

Fig. 1. Predominant colonic micro-organisms categorized into potentially harmful or health-promoting groups (modified from Gibson \& Roberfroid, 1995).

putrefaction, whereas health-promoting effects may be derived from the inhibition of growth of harmful bacteria, stimulation of immune functions, lowering of gas distention problems, improved digestion/absorption of essential nutrients and systemic effects on blood lipids. Although these benefits have not been well proven in humans and therefore remain largely speculative, it may be possible to categorize the gut microbiota components on the basis of whether they exert potentially pathogenic or health-promoting aspects (Fig. 1). Attempts to stimulate micro-organisms that carry out the latter may have obvious benefits. As diet forms a major determinant of gut bacterial activities, this would mainly be achieved through ingestion of certain foodstuffs. Most attention in this respect has been given to the use of probiotics as live microbial feed supplements (Fuller, 1992, 1997). However, it may also be the case that non-viable dietary components stimulate the potentially beneficial microbiota.

\section{Colonic fermentation}

The principal substrates for bacterial growth are dietary carbohydrates that have escaped digestion in the upper gastrointestinal tract. These include resistant starch, nonstarch polysaccharides, and unabsorbed sugars, oligosaccharides, food additives and sugar alcohols. There is also a contribution from endogenous carbohydrates like mucins and chondroitin sulphate. In addition, both dietary and endogenously derived proteins/amino acids can be utilized for growth.

Principal endproducts of bacterial fermentation in the colon are short chain fatty acids (SCFA) such as acetate. propionate and butyrate. $\mathrm{A}$ number of gases are also produced, including $\mathrm{H}_{2}, \mathrm{CO}_{2}, \mathrm{H}_{2} \mathrm{~S}$ and $\mathrm{CH}_{4}$. Fermentation intermediates produced in the colon include ethanol, lactate, succinate and pyruvate and these may be further fermented to SCFA which allows further energy gain for the host from fermentation. Proteolytic species may cause an accumulation of endproducts such as ammonia. phenolic compounds and amines, whilst amino acid metabolism yields branched chain fatty acids.

The composition of an individual's colonic microbiota is usually considered to be fairly stable over long periods. However, a number of physicochemical factors can influence the pattern and extent of fermentation of particular substrates. These include competition for 
nutrients, physicochemical environment of the large gut, metabolic interactions between bacteria and dietary variances (Holdeman et al. 1976; Simon \& Gorbach, 1982; Macfarlane \& Gibson, 1994; Gibson \& Macfarlane, 1995).

There is currently a great deal of interest in manipulation of the composition of the gut flora towards a potentially more healthy community. That is, an increase in numbers and activities of bacterial groups which have healthpromoting properties (like Bifidobacterium, Lactobacillus) is desirable. This should be possible by the use of dietary adjuncts which reach the colon intact and may be specifically fermented. Such materials are termed prebiotics (Gibson \& Roberfroid, 1995).

\section{Prebiotics - dietary components that are specifically fermented in the hindgut}

A prebiotic is 'a non-digestible food ingredient that beneficially affects the host by selectively stimulating the growth and/or activity of one or a limited number of bacteria in the colon, that can improve the host health' (Gibson \& Roberfroid, 1995). Whilst any food ingredient that enters the large intestine is possibly a prebiotic, it is selectively of the fermentation, in the mixed bacterial environment, that is critical and required (Fig. 2). At present, most prebiotics are selected on the basis of their ability to promote the growth of lactic acid producing micro-organisms.

Most success has been derived from oligosaccharides containing fructose. Figure 2 shows how oligofructose (degree of polymerization $=4$ ) and inulin (degree of polymerization $=10$ ) may alter the gut microbiota composition in controlled volunteer trials (Gibson et al. 1995). Other studies have confirmed the bifidogenic nature of these oligosaccharides both in vitro and in vivo (e.g. Hidaka et al. 1986; Buddington et al. 1996; Kleesen et al. 1997).

Various other oligomers may also be prebiotic. These include some sugar alcohols, lactulose, as well as oligosaccharides that contain xylose, mannose, galactose, soya, maltose and mannose.

Important developments also include the use of prebiotics that have activities with multiple biological functions. This would clearly involve stimulation of potentially beneficial micro-organisms, such as the lactic acid microflora. However, further possibilities exist for the use and manufacture of prebiotics that incorporate more than one activity. Here, the anti-infective nature of fermentable gut substrates may be effective through blocking adhesion site that may otherwise be occupied by pathogens (Zopf \& Roth, 1996). For example, oligosaccharides that have mannose

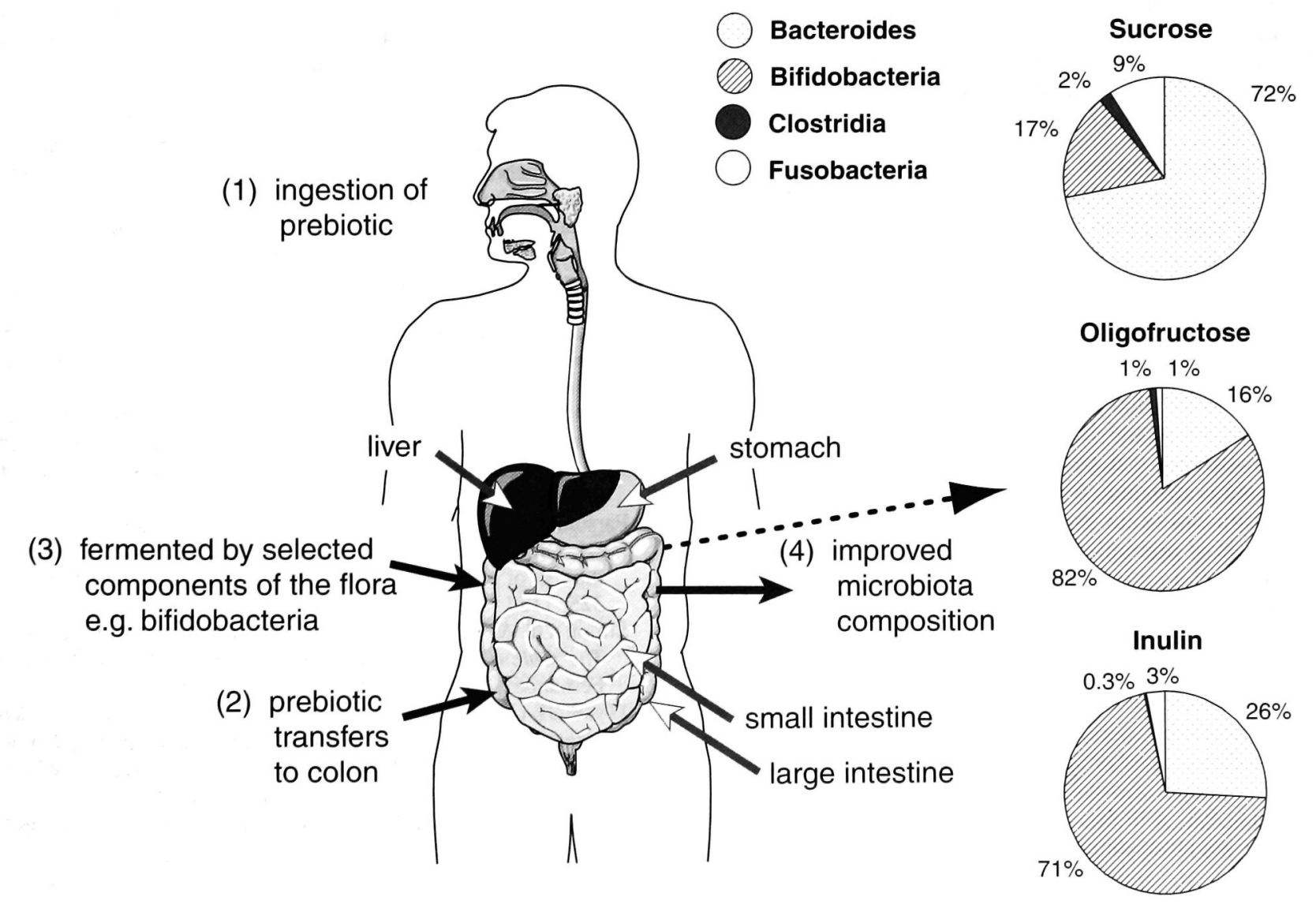

Fig. 2. The prebiotic concept. Pie diagrams show how the microflora can develop during the feeding of $15 \mathrm{~g} / \mathrm{d}$ sucrose, oligofructose and inulin the fructo-oligosaccharides acting as prebiotics by stimulating bifidobacteria. Data from Gibson et al. (1995). 
side chains may be effective against Escherichia coli and related organisms.

There may also be a possibility that oligosaccharides which offer 'natural' attenuative properties (such as the known effects of cellobiose against virulence properties in Listeria monocytogens; Park \& Kroll, 1993) prove to be a further important biological function. The enzymatic synthesis of 'designer' molecules with multiple activities is a research avenue of much promise, as is the derivation of oligosaccharides from fibre- and starch-containing polymers. For prebiotics, it is clear that the future potential will not rest solely with carbohydrates extracted from certain foodstuffs, e.g. fructo-oligosaccharides. In terms of new prebiotic development, the following aspects may be worthy of research considerations:

(1) incorporation into appropriate food vehicles;

(2) stimulate a beneficial flora but also exert anti-adhesive as well as attenuative properties;

(3) low dosage forms;

(4) derive from dietary polysaccharides;

(5) non-cariogenic;

(6) good preservative and drying characteristics;

(7) viscosity regulation;

(8) low calorific value.

Whilst it is encouraging that many different possible prebiotics exist, the future potential will only be realized by exploiting the latest research methodologies. For example, a molecular approach to the gut fermentation will increasingly attract genetic fingerprinting and other highly efficacious applications to (anaerobic) human gut bacteriology. This includes:

(1) restriction fragment length polymorphism (e.g. pulsed field gel electrophoresis, robotyping, amplified fragment length polymorphism);

(2) direct amplification (using polymerase chain reaction);

(3) the design of specific gene probes.

\section{Conclusion}

The microbiota of the human gastrointestinal tract is key for the nutrition and health of the host, but may contain harmful, benign and beneficial micro-organisms - all of which may respond to dietary ingredients. Microflora modulation can occur through foodstuffs that contain sufficient levels of prebiotics that target the (potentially) healthpromoting flora. Future research on the development of prebiotic ingredients/foodstuffs, the tracking of microflora changes using modern molecular methodologies and definitive assessments of the health bonuses offer great potential.

\section{References}

Buddington RK, Williams CH, Chen SC \& Witherly SA (1996) Dietary supplementation of neosugar ilters the fecal flora and decreases activities of some reductive enzymes in human subjects. American Journal of Clinical Nutrition 63, 709-716.

Cummings JH \& Macfarlane GT (1991) A review: the control and consequences of bacterial fermentation in the human colon. Journal of Applied Bacteriology 70, 443-459.

Dixon MF (1992) Helicobacter pylori and chronic gastritis. In Helicobacter pylori and Gastroduodenal Disease, 2nd edn, pp. 124-139 [BJ Rathbone \& RV Heatley, editors]. Oxford: Blackwell Scientific Publications.

Finegold SM, Sutter VL \& Mathisen GE (1983) Normal indigenous intestinal flora. In Human Intestinal Microflora in Health and Disease, pp. 3-31 [DJ Hentges, editor]. London: Academic Press.

Fuller R [editor] (1992) Probiotics: The Scientific Basis. London: Chapman \& Hall.

Fuller R [editor] (1997) Probiotics 2: Applications and Practical Aspects. London: Chapman \& Hall.

Gibson GR, Beatty EB, Wang X \& Cummings JH (1995) Selective stimulation of bifidobacteria in the human colon by oligofructose and inulin. Gastroenterology 108, 975-982.

Gibson GR \& Macfarlane GT [editors] (1995) Human Colonic Bacteria. Role in Physiology, Pathology and Nutrition. CRC Press, Boca Raton.

Gibson GR \& Roberfroid MB (1995) Dietary modulation of the human colonic microbiota: introducing the concept of prebiotics. Journal of Nutrition 125, 1401-1412.

Hidaka H, Eida T, Takiwaza T, Tokunga T \& Tashiro Y (1986) Effects of fructooligosaccharides on intestinal flora and human health. Bifidus Microflora 5, 37-50.

Holdeman LV, Good IJ \& Moore WEC (1976) Human fecal flora: variation in bacterial composition within individuals and a possible effect of emotional stress. Applied Environmental Microbiology 31, 359-375.

Kleessen B, Sykura B, Zunft H-J \& Blaut M (1997) Effects of inulin and lactose on fecal microflora, microbial activity, and bowel habit in elderly constipated persons. American Journal of Clinical Nutrition 65, 1397-1402.

Macfarlane GT \& Gibson GR (1994) Metabolic interactions between colonic bacteria and the host. In Human Health: the Contribution of Microorganisms, pp. 17-52 [SAW Gibson, editor]. London: Springer-Verlag.

Moore WEC \& Holdeman LV (1974) Human fecal flora: the normal flora of 20 Japanese-Hawaiians. Applied and Environmental Microbiology 27, 961-979.

Park SF \& Kroll RG (1993) Expression of listeriolysin and phosphatidylinositol-specific phospholipase $\mathrm{C}$ is repressed by the plant-derived molecule cellobiose in Listeria monocytogenes. Molecular Microbiology 8, 653-661.

Parsonnet J, Friedman GD, Vandersteen DP, Chang Y, Vogelman JH, Orentreich N \& Sibley RK (1991) Helicobacter pylori and the risk of gastric carcinoma. New England Journal of Medicine 325, 1127-1131.

Simon GL \& Gorbach SL (1982) Bacteriology of the colon. In The Colon: Structure and Function, pp. 103-114 (L BustosFernandez, editor]. London: Plenum Medical.

Wyatt JI (1992) Helicobacter pylori, duodenitis and duodenal ulceration. In Helicobacter pylori and Gastroduodenal Disease, 2nd edn, pp. 140-149 [BJ Rathbone \& RV Heatley, editors]. Oxford: Blackwell Scientific Publications.

Zopf D \& Roth S (1996) Oligosaccharide anti-infective agents. Lancet 347, 1017-1021. 